\title{
Gallbladder Tumor Treated Through Chemotherapy: A Case Report
}

ISSN: 2637-7632

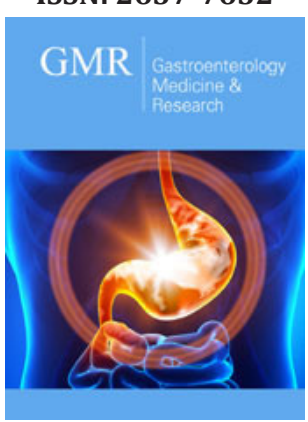

*Corresponding author: João Kleber de Almeida Gentile, Medical Professor of the Discipline of Digestive Surgery in University City of São Paulo (UNICID). Medical doctor and Resident Physician, Department of Digestive Surgery, Hospital do Servidor Público Municipal (HSPM-SP), São Paulo (SP), Brazil

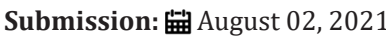

Published: 温 August 18, 2021

Volume 6 - Issue 1

How to cite this article: Emanuela Alves Delmondes, Júlia Guimarães Pereira, João Kleber de Almeida Gentile, et al. Gallbladder Tumor Treated Through Chemotherapy: A Case Report. Gastro Med Res. 6(1). GMR. 000630. 2021.

DOI: 10.31031/GMR.2021.06.000630

Copyright@ João Kleber de Almeida Gentile, This article is distributed under the terms of the Creative Commons Attribution 4.0 International License, which permits unrestricted use and redistribution provided that the original author and source are credited.
Emanuela Alves Delmondes ${ }^{1}$, Júlia Guimarães Pereira ${ }^{1}$, Lais de Campos Bento ${ }^{1}$, Mariana Mussalem Santos ${ }^{1}$, Natália dos Santos Ramalho Vieira1, Renato Migliore $^{2}$, João Kleber de Almeida Gentile ${ }^{2 *}$, Rodrigo Biscuola Garcia ${ }^{3}$, Pedro Marcos Santinho Bueno de Souza ${ }^{3}$ and Jose Cesar Assef ${ }^{4}$

${ }^{1}$ Graduate of Medicine in University City of São Paulo (UNICID), São Paulo (SP), Brazil

${ }^{2}$ Medical Professor of the Discipline of Digestive Surgery in University City of São Paulo (UNICID). Medical doctor and Resident Physician, Department of Digestive Surgery, Hospital do Servidor Público Municipal (HSPM-SP), São Paulo (SP), Brazil

${ }^{3}$ Medical doctor and Attending Physician, Department of Digestive Surgery, Hospital do Servidor Público Municipal (HSPM-SP), São Paulo (SP), Brazil

${ }^{4}$ Medical doctor and Chief, Department of Digestive Surgery, Hospital do Servidor Público Municipal (HSPM-SP), São Paulo (SP), Brazil

\begin{abstract}
The Gallbladder neoplasia is rare and has a high mortality rate, thus surgery is still the usual curative treatment for the disease. Once its diagnosis is usually tricky and at a late stage, there is the necessity of using neoadjuvant chemotherapy in advanced cases. We report the case of a 51-year-old man diagnosed with gallbladder cancer presenting liver invasion but no metastasis. Initially, it was considered inoperable so the patient was submitted to neoadjuvant chemotherapy with gemcitabine. By the end of chemotherapy, the radiological examination showed the complete reduction of the lesion, choosing to proceed with the salvage surgery. Therefore, it was performed conventional cholecystectomy with liver resection (segments V and VI) and regional lymphadenectomy. The histopathological examination did not show neoplasia in any of the resected tissues or lymph nodes involvement. The patient evolved to the cure of the disease with chemotherapy treatment. Six years after the primary diagnosis, the patient remains being monitored with no recurrence of the tumor.
\end{abstract}

\section{Introduction}

Gallbladder cancer is a rare but highly malignant disease in countries such as India, Chile, and Japan, where it has a high mortality rate [1,2]. Due to its low incidence in most Western countries, it has been little studied, leading to variations in the approaches of the initial pathological evaluation, classification and staging of the disease [3]. Surgery is the most effective and potentially curative treatment for gallbladder cancer. However, most patients have locally advanced disease, due to the lack of initial symptoms, and survival for these patients remains very low. In patients with gallbladder cancer receiving palliative therapy, the median survival time was about 6 months $[4,5]$. Therefore, there is a clear need for effective chemotherapeutic methods in the treatment of gallbladder cancer. Recently, new therapeutic regimens have been approved for the treatment of bile duct cancer, which include cisplatin and gemcitabine [6]. We report a case of a patient with advanced gallbladder neoplasm undergoing chemotherapy treatment with cure of the primary disease.

\section{Case Report}

A 51-year-old man went to the hospital due to abdominal pain in the right hypochondrium and jaundice was diagnosed for advanced gallbladder neoplasia being referred for 
investigation and clinical staging in February 2012. Initial admission data showed a discrete increase in total serum bilirubin (Gammaglutamyltranspepticity ( $\gamma$-GGT 187IU/L) and carcinoembryonic antigen (CEA 18ng/dL). Other laboratory tests and tumor markers were within normal range. Initially computed tomography (CT) of the abdomen revealed a hypodense, heterogeneous mass with imprecise limits, measuring $97 \times 58 \times 76 \mathrm{~mm}$ (volume $=225 \mathrm{~cm}^{3}$ ) with projection to the gallbladder bed, affecting segments $\mathrm{V}$ and VI of the liver, without presenting dilatation of the intra- or extrahepatic viliary pathways suggesting a primary neoplastic process of the gallbladder (Figure 1). MRI of the abdomen presented similar findings without evidence of hepatic or distant metastases. Percutaneous biopsy of the CT guided lesion was performed, confirming the presence of gallbladder adenocarcinoma confirmed by the immunohistochemical pattern. The diagnosis of undifferentiated gallbladder adenocarcinoma with hepatic invasion was then considered, considering the patient initially inoperable and then referred to the oncology for neoadjuvant treatment.

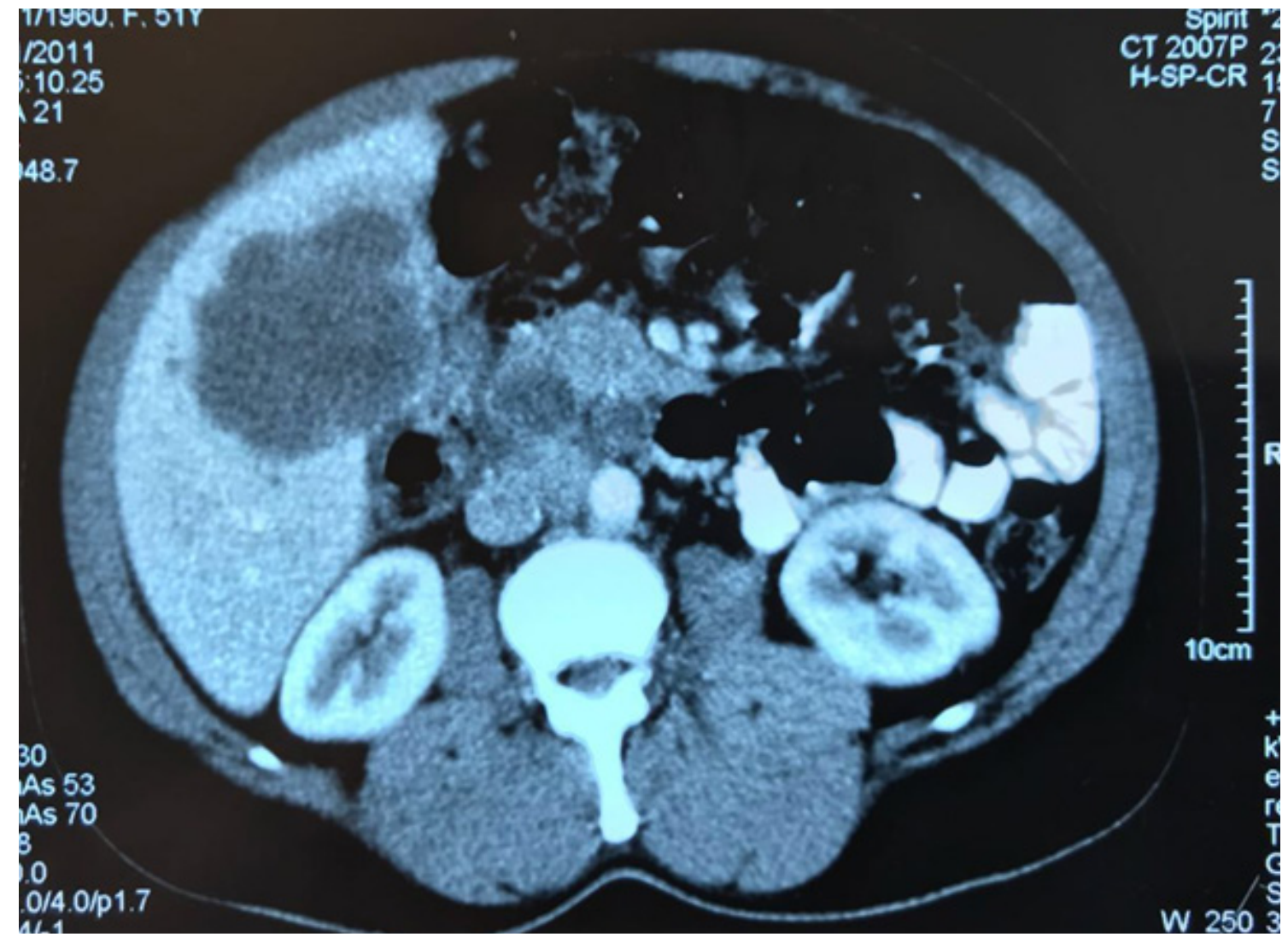

Figure 1: CT axial abdomen showing a hypodense and heterogeneous mass, measuring $97 \times 58 \times 76 \mathrm{~mm}$ $\left(\right.$ volume $=225 \mathrm{~cm}^{3}$ ) with projection to the gallbladder bed affecting the V and VI segments of the liver, suggesting a primary neoplastic process of the gallbladder.

With the consent of the patient chemotherapy with gemcitabine was introduced at the dose of $1000 \mathrm{mg} / \mathrm{m}^{2}$ weekly for 3 weeks, followed by a rest of 1 week and the schedule was repeated on an outpatient basis. His serum CEA level decreased gradually, and at follow-up CT for re-staging performed 8 cycles later in June 2012, showed reduction of gallbladder and hepatic gallbladder injury. In November 2012, 12 cycles later new CT scan showed complete reduction of the visible lesion in the initial CT and was then chosen for salvage surgery (Figure 2). Macroscopically no lesion was identified as well as peritoneal dissemination or distant metastases, with conventional cholecystectomy with resection of the hepatic bed (segments V and VI) and regional lymphadenectomy. Microscopically, there was no evidence of neoplasia in any resected organ or even in the lymph nodes, which was followed by a rigorous revision of the slides by the pathology team, showing complete healing of the lesion with the chemotherapeutic scheme. 


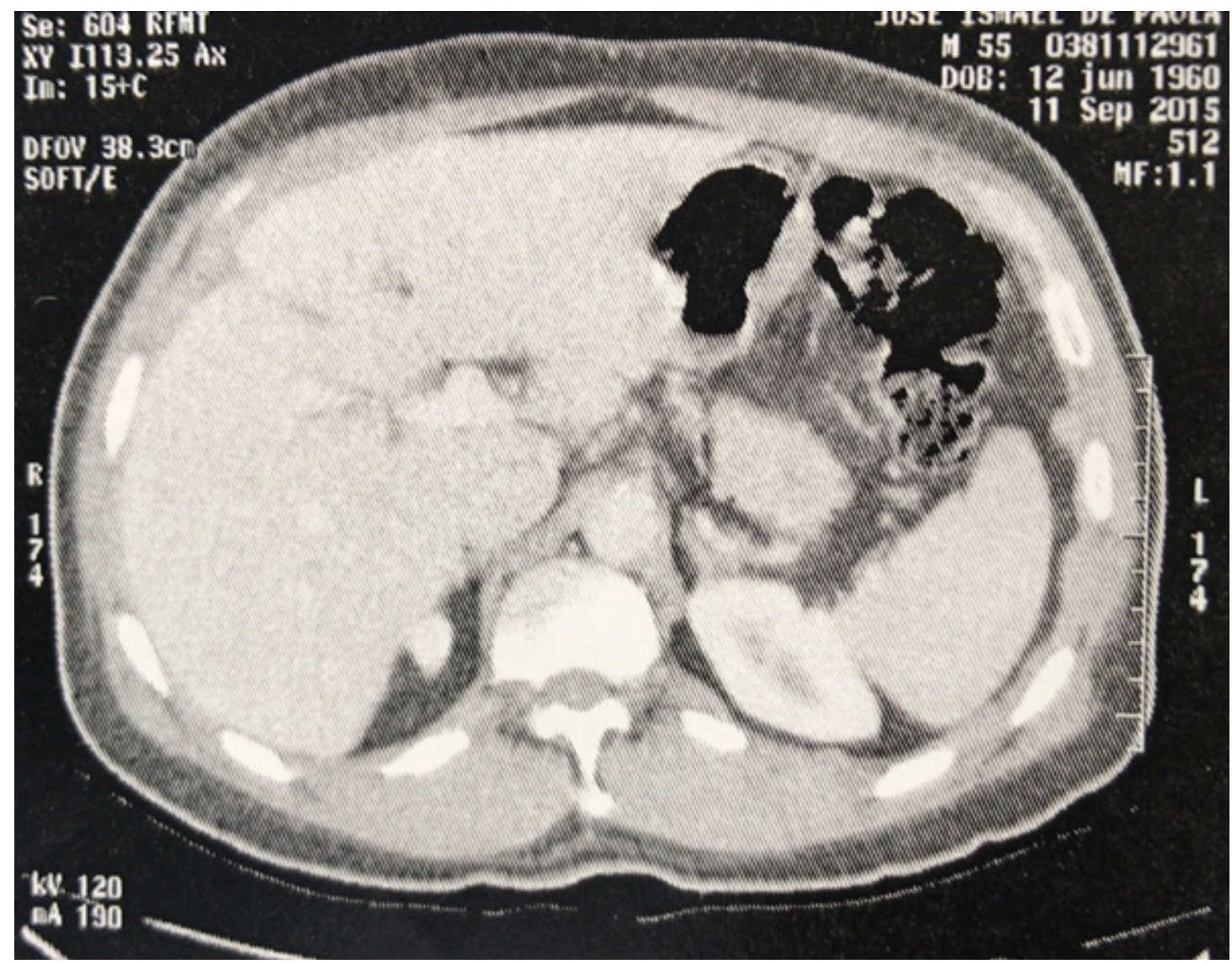

Figure 2: CT axial abdômen showing complete regression of hepatic lesion and gallbladder without evidence of locoregional involvement.

The patient progressed with a favorable post-operative course and was maintained without adjuvant chemotherapy. At present, 6 years after the initial diagnosis, the patient presents with no evidence of tumor recurrence, with a serum level of CEA 2 [7]. being followed up every six months.

\section{Discussion}

More than $90 \%$ of gallbladder cancers are well-differentiated adenocarcinomas $[8,9]$. They are associated with a 5-year survival rate of 5-13\% [5]. Most patients have locally advanced disease due to late diagnosis, lack of symptoms initiating a poor prognosis [6]. It also does not present established chemotherapy protocols due to the lack of curative evidence with chemotherapeutic agents and because the surgical therapy has been considered as the only potential cure. However, there are currently several lines of research on the efficacy of associated or isolated chemotherapy, aiming to increase survival $[6,10]$. Although studies have shown that the combination of gemcitabine with other chemotherapeutics has a greater benefit in increasing patient survival, the use of gemcitabine alone provides a significant prolongation of life expectancy. Okusaka, et al. [11] 2010 shows that the gallbladder cancer control rate is $68.3 \%$ with the use of gemcitabine and cisplatin is $50 \%$ with gemcitabine alone, proving that there is efficacy of gemcitabine monotherapy [12]. Penz et al. [12] shows that the use of gemcitabine alone in high doses allowed stabilization of the disease in $44 \%$ of patients, with an average time of 11.5 months of survival; in agreement with other studies that report an increase in survival with the use of this chemotherapeutic drug [13]. Reports that show cure of gallbladder cancer with the use of chemotherapy are scarce $[13,14]$. But we can state, according to our patient's response to chemotherapy, the effectiveness of the use of neoadjuvant gemcitabine as a curative treatment after a total of 12 cycles.

\section{Conclusion}

Cancer of the gallbladder is an uncommon disease, difficult to treat and no established chemotherapy protocols for its management. However, there are several lines of research on the efficacy of associated or isolated chemotherapy, aiming at increasing survival. In the case reported, the effect of gemcitabine alone was shown in the complete remission of gallbladder adenocarcinoma and its projection for the V and VI segments of the liver; resulting in its complete healing.

\section{References}

1. Goldin RD, Roa JC (2009) Gallbladder cancer: A morphological and molecular update. Histopathology 55(2): 218-229.

2. Albores-Saavedra J, Kl€oppel G, Adsay N, Sripa B, Crawford J, et al. (2010) Carcinoma of the gallbladder and extrahepatic bile ducts. In: Bosman F, Carneiro F, Hruban R (eds.), World Health Organization Classification of Tumours of the Digestive System. IARC Press, Lyon, France, pp. 266-274. 
3. Frierson HF Jr (1989) The gross anatomy and histology of the gallbladder extrahepatic bile ducts, Vaterian system, and minor papilla. Am J Surg Pathol 13(2): 146-162.

4. limelius B, Hoffman K, Sjödén PO, Jacobsson G, Sellström H, et al. (1996) Chemotherapy improves survival and quality of life in advanced pancreatic and biliary cancer. Ann Oncol 7(6): 593-600.

5. Ishikawa T, Horimi T, Shima Y, Okabayashi T, Nishioka Y, et al. (2003) Evaluation of aggressive surgical treatment for advanced carcinoma of the gallbladder. J Hepatobiliary Pancreat Surg 10(3): 233-238.

6. McNamara MG, Metran-Nascente C, Knox JJ (2013) State-of-the-art in the management of locally advanced and metastatic gallbladder cancer. Curr Opin Oncol 25(4): 425-431.

7. Goldin R, Roa J (2009) Gallbladder cancer: A morphological and molecular update. Histopathology 55(2): 218-229.

8. Okumura T, Nakamura J, Kai K, Ide Y, Nakamura H, et al. (2014) Curative resection of gallbladder cancer with liver invasion and hepatic metastasis after chemotherapy with gemcitabine plus S-1: Report of a case. World Journal of Surgical Oncology 12(1): 326.

9. Lazcano-Ponce E, Miquel J, Munoz N, Herrero R, Ferrecio C, et al. (2001) Epidemiology and molecular pathology of gallbladder cancer. CA Cancer J Clin 51(6): 349-364.
10. Sugita H, Hirota M, Ichihara A, Furuhashi S, Kihara S, et al. (2006) Combined chemotherapy of irinotecan and low-dose cisplatin (I/ low-P) against metastatic biliary tract cancer. Journal of Hepato-BiliaryPancreatic Surgery 13(5): 463-467.

11. Okusaka T, Nakachi K, Fukutomi A, Mizuno N, Ohkawa S, et al. (2010) Gemcitabine alone or in combination with cisplatin in patients with biliary tract cancer: A comparative multicenter study in Japan. British Journal of Cancer 103(4): 469-474.

12. Penz C, Kornek G, Raderer M, Ulrich-Pur H, Fiebiger W, et al. (2001) Phase II trial of two-weekly gemcitabine in patients with advanced biliary tract cancer. Annals of Oncology 12(2): 183-186.

13. Morimoto H, Ajiki T, Takase S, Fujita T, Matsumoto T, et al. (2008) Resection of gallbladder cancer with hepatic metastasis after chemotherapy with gemcitabine. Journal of Hepato-Biliary-Pancreatic Surgery 15(6): 655-658.

14. Okumura T, Nakamura J, Kai K, Ide Y, Nakamura H, et al. (2014) Curative resection of gallbladder cancer with liver invasion and hepatic metastasis after chemotherapy with gemcitabine plus S-1: report of a case. World Journal of Surgical Oncology 12(1): 326.

For possible submissions Click below: 